\title{
Role of genetic and molecular predictors in optimization of in vitro fertilization programs for selective embryo transfer
}

\begin{abstract}
Introduction: This research presents data about embryo capability for implantation based on the study of changes in metabolomic profile of embryo culture media, consumption of constituents of embryo culture media and anamnestic factors predisposing to aneuploid embryos. Determination of glucose and glutamate concentrations in spent media samples from embryos collected on day 5 is a new noninvasive method of assessment embryo quality to efficiency improvement of IVF outcomes.
\end{abstract}

Material and methods: 96 couples underwent IVF program with preimplantation genetic testing (PGT). Spent media samples from embryos that resulted in pregnancy and delivery and samples from embryos that failed to implant were individually collected on day 5, and evaluated using mass-spectrometry.

Results: Correlation was identified between metabolomic profile, implantation potential of embryo and ploidy of embryos.

Conclusions: Analysis of composition of embryo culture media maid by method of metabolomic profiling can result in more specific selection of embryos for further transfer.
Volume 10 Issue $3-2019$

\author{
Veronika Smolnikova,' Inna Zorina,' Michael \\ Bobrov, ${ }^{1,2}$ Elena Kalinina,' Natalia Makarova,' \\ Ibragimova Luiza,' Suhih Gennady' \\ IIVF, Kulakovs Scientific Centre of Obstetrics, Gynecology and \\ perinatology, Russia \\ ${ }^{2}$ Oxylipin lab, Shemyakin-Ovchinnikov Institute of Bioorganic \\ Chemistry, Russia
}

Correspondence: Veronika Smolnikova, IVF, Kulakovs Scientific Centre of Obstetrics, Gynecology and perinatology, Moscow, Russia, Tel +7 (903)723 08 47,

Email veronika.smolnikova@mail.ru

Received: June 07,2019 | Published: June 28, 2019

\section{Introduction}

Problems with conception are still relevant for couples of reproductive age throughout the world. For some of them, the only chance to have a child is using assisted reproductive technologies (ART).

There are many factors which effect success of infertility treatment: ovarian reserve, patient age, infertility factor, concomitant gynecological and somatic diseases, timely detection of intrauterine pathology, endometrial morphology, determination of the implantation window.

Implementation of special methods of conception, constant improvement of drugs, personalized guideline-based treatment recommendations, modification of embryo culture methodology can lead to higher rate of successful treatment of infertility. However, this does not solve the problem of unsuccessful outcomes of IVF programs. Pregnancy rate does not exceed $50 \%$ even if woman has euploid embryo and normal characteristics of the endometrium before the embryo transfer (the endometrium thickness exceeds $8 \mathrm{~mm}$ ). ${ }^{1,2}$

Assessing the morphological quality of embryos is a common method of selecting an embryo suitable for transfer. Embryologists use the blastocyst scoring system developed by Gardner et al.

This grading system has three separate quality scores for each blastocyst. The final score assigned for each blastocyst is composed of these three scores. Therefore the first number is the expansion score, a number from 1-6 based on degree of expansion and the hatching status. The ICM score is listed second as, A. many cells forming a cohesive epithelium, B. few cells forming a loose epithelium and C. very few large cells. The final score is the TE score (A. tightly packed, many cells, B. loosely grouped, several cells and C. very few cells). ${ }^{3-5}$

On the one hand there is always a risk that good quality embryos would not implant because they have aneuploid chromosome number. ${ }^{6}$
On the other hand poor quality embryos can lead to pregnancy and increase the number of live births. ${ }^{6}$

The world's largest studies reported that $10 \%$ cases of termination of pregnancy related to chromosomal abnormalities:

chromosomes either missing from or extra to the normal pair. ${ }^{7-12}$ For the last 10 years Preimplantation genetic testing (PGT) has been reliable method of diagnosticating genetic abnormalities of embryos before transferring them to the uterine cavity. ${ }^{13}$ The rates of successful implantation, live birth have increased recently while the number of miscarriages of patients with PGD has declined in comparison to patients with standard IVF. ${ }^{14-16}$ Despite the fact that such method of embryo selection improves morphological quality there are certain disadvantages that should be mentioned. Such as chance of negative influence to embryo during trophectoderm biopsy, of mosaicism during interpretation of results, chance of self correction and selective apoptosis of embryo on early stages of development and also high cost of research and inability to provide research in lack of material. ${ }^{17}$ Besides PGD cannot completely exclude a chance of existence of genetic numerical aberration and point mutation, that could affect implication of embryo and lead to development of congenital malformation. ${ }^{18-23}$

That is why improvement of modern approaches to investigation of biological processes that impair implantation ${ }^{24-26}$ and non-invasive diagnostic techniques of viability evaluation of embryos are of great importance. ${ }^{27-29}$

Omics technologies are new spheres of science allowing to investigate functioning of cellular structures from DNA and genes to Metabolites. Application of omics technologies helps to detect new molecular infertility biomarkers that lead to widening of diagnostic capability and improving IVF results.

Embryo culture medium containing information about metabolic activity, energy metabolism and condition of cell signaling pathway 
is unique subject of study. ${ }^{30} \mathrm{In}$ view of the aforesaid it is relevant and promising to find out the predictors of successful implantations leading to pregnancy and live birth.

The Goal of research is optimization of ART methods by determining ability of embryo to successful implantation during selective embryo transfer to uterine cavity. On the bases of investigation of metabolomic profiles, namely glucose and glutamate consumption by embryo culture medium.

\section{Material and methods}

Retrospective and prospective study of 96 married couples who applied for an IVF-program with PGT. All patients were previously examined. Morphological assessment was performed on day 5 and then trophectoderm biopsy of blastocyst for PGD by using comparative genomic hybridization method (aCGH). The time needed to obtain the results of the genetic tests may not allow a fresh embryo transfer to be performed. The blastocysts cryopreserved immediately after the biopsy. To determine molecular genetic quality predictors of developing embryos, all samples of culture media were conveniently classified into groups depending on their genetic and morphological quality and outcomes of embryo transfer to the uterine cavity. Metabolic profiles of spent culture media were analyzed using massspectrometry

\section{Entry criteria}

a. IVF programs using PGD

b. age from 18 to 45

c. Normal ovarian reserve (FSH levels measured on day 3 of the menstrual cycle $\leq 10 \mathrm{IU} / \mathrm{L}$; AMH not less than $1.0 \mathrm{ng} / \mathrm{ml}$; antral follicle count (AFC) not less than 5 in each ovary according to ultrasound)

d. normal anatomy of uterine

e. Normal results of karyotyping of both partners $(46, \mathrm{XX} ; 46, \mathrm{XY})$

f. Signed patient informed consent

\section{Non-inclusion criteria}

A. Female reproductive system pathology:

a. congenital defects, excluding the possibility of embryo implantation and carrying of a pregnancy

b. Stage III-IV endometriosis, large uterine leiomyomas, ovarian tumors, Polycystic ovary syndrome (PCOS), diminished ovarian reserve, surgical interventions on the ovaries and etc.

B. Other diseases that can be counterindications for carrying of a pregnancy

a. somatic diseases: thyroid gland diseases, acute inflammatory diseases, exacerbation of chronic diseases.

b. mental disorder

c. Recent malignant tumor any location or mentioned in past medical history

C. Severe form of pathozoospermia

\section{Exclusion criteria}

a) Absense of follicular growth during ovarian stimulation

b) Absense of oocytes during transvaginal ultrasound-guided oocyte retrieval (TVOR)

Ovarian stimulation begins on day 2-5 of the menstrual cycle according to protocol with gonadotropins and gonadotropin-releasing hormone antagonist (GnRH antagonists) with further PGD by using comparative genomic hybridization method (aCGH)

Program was conducting according to standard protocols with gonadotropins and gonadotropin-releasing hormone antagonist depending to age of patient, ovarian reserve and hormonal state.

After $35 \mathrm{~h}$ of HCG injection (dosage 10 000IU on follicular size$18 \mathrm{~mm}$ or greater in maximal diameter.) oocytes were collected by transvaginal ultrasoundguided needle aspiration of the follicles under deep conscious sedation. For fertilization was used method ICSI to avoid effect of side DNA. On 5 day of cultivation after morphological analysis (scoring system developed by Gardner et al) embryos' trophectoderm was biopsied and then cryopreservation of embryos is performed.

Metabolic profiles of spent culture media were investigated by mass-spectrometry to identify potential biomarkers of successful implantation.

\section{Results}

In order to reveal factors effecting morphological quality of embryos in IVF programs method of logistic regression was used. Dichotomous variable was morphological quality of embryo either good or fair. According to logistic regression coefficients should not be correlated. In case of correlation coefficient is excluded.

Multifactorial allowed to determine influence of independent factors on dependent variable. As independent further factors were determined: age, menarche, regular menstrual cycle, BMI, anamnestic data, factor of infertility, quantity of IVF programs (ICSI), dosage of drugs and duration of stimulation, method of fertilization, quality of embryos and karyotype.

Step by step inclusion and exclusion of above mentioned variables allowed determining exact predictors effecting the result.

There were 306 PGT 56,9\% (174) of them were of good morphological quaility, 37,9\% (116) were of fair morphological quaility and 5,2 (16) were of poor morphological quaility. In groups of embryos with good quality $40 \%$ (69) were with chromosomal abnormalities. In groups of embryos with fair quality $62 \%$ (72) were with chromosomal abnormalities and in groups of embryos with poor quality $23 \%$ (69) were with chromosomal abnormalities. In other words there were less aneuploid embryos after PGT in group of good morphological quality than in group fair morphological quality.

Within the patient group of under 34 years 172 embryo were examined for genetic abnormalities, $61 \%$ had euploid karyotype. 36 embryos were taken from patients of 35-39 years and $47 \%$ of them have euploid karyotype (17). 98 embryos were taken from patients over 40 years old and $40 \% \mathrm{f}$ them have euploid karyotype (40). It is obvious that there is direct correlation between age and ploidy of embryos. 
Besides the correlation between wrong genetic embryo karyotype and external genital endometriosis and also type of infertility was revealed.

Taking into account change of composition of materials during research all profiles of spent culture media metabolites were divided in two groups - intraspecific and cross-species.

On 5 day of cultivation all embryos were divided in 4 classes according to morphological research and given morphological mark. Principal components analysis (PCA) was used to reduce size of multicomponent selections, data and for visualiztion of clusterization examples according to metabolites profile. Differences in profiles spent culture medium metabolites were revealed.

It is remarkable that all above mentioned classes were different to group of controlled culture medium (without cultivation of embryos) While embryos that stoped growing at early stages had no differences to controlled group

Intraspecific comparison of embryos of one morphological class did not show any differences between metabolomic profiles. Just as well profiles of culture media of euploid and aneuploid embryos. While metabolomic profiles of different morphological classes have variations

At the next stage, the analysis of the correlations between levels of metabolites in the spent culture media of patients after IVF(ICSI) program with PGD included in the study.

PCA allowed detecting significant differences between groups with different outcomes after selective embryo transfer. Compounds were identified by chromographic retention time, molecular mass and fragmentation of the corresponding molecular ion.

With a qualitative comparative analysis of the parameters of the obtained mass peaks in spent culture media of implanted embryos concentration of L-Valine (118,0864 Da), L-Proline (138,0526 Da), alanyn-glutamine $(218,1144 \mathrm{Da})$, Phenylpyruvic acid ( 165,0545 Da) и b-L-Fucosa-1(166,0866 Da) has decreased, and concentration of 1-phenylalanine $(166,0866 \mathrm{Da})$ has increased by comparison to spent culture media of unimplanted embryos. This result suggests that changes in embryo metabolism at the cultivation stage have significant importance for embryo implantation (Table 1).

Table I Changes in the concentration of the investigated substances in the culture media of implanted embryos compared with non-implanted

\begin{tabular}{lll}
\hline $\begin{array}{l}\text { Molecular } \\
\text { mass, Da }\end{array}$ & $\begin{array}{l}\text { Presumed } \\
\text { molecule }\end{array}$ & $\begin{array}{l}\text { Concentration } \\
\text { changes }\end{array}$ \\
\hline I I8.0864 & L - valin & I8 times less \\
I38.0526 & L- proline & 4100 times less \\
218.1144 & alanyn-glutamine & 19 times less \\
I66.0866 & L-phenylalanine & 287 times more \\
165.0545 & Phenylpyruvic acid & 697 times less \\
267.0257 & b-L-Fucosa-I & 3069 times less \\
\hline
\end{tabular}

Moreover during this research it was found out that glucose consumption level by first class embryos 1,75 times more than that of second class embryos and two times more than of third class embryos. There were no differences connected to this parameter between second and third class of embryos.

There is no reliable data about differences in glutamate consumption between embryos of all classes.
PGT showed that at average $50 \%$ of investigated in research embryos have aneupoloud karyotype.

Intraspecific comparison revealed no differences between euploid and aneuploid embryos in consumption of glucose and glutamate (Figure 1) (Figure 2).

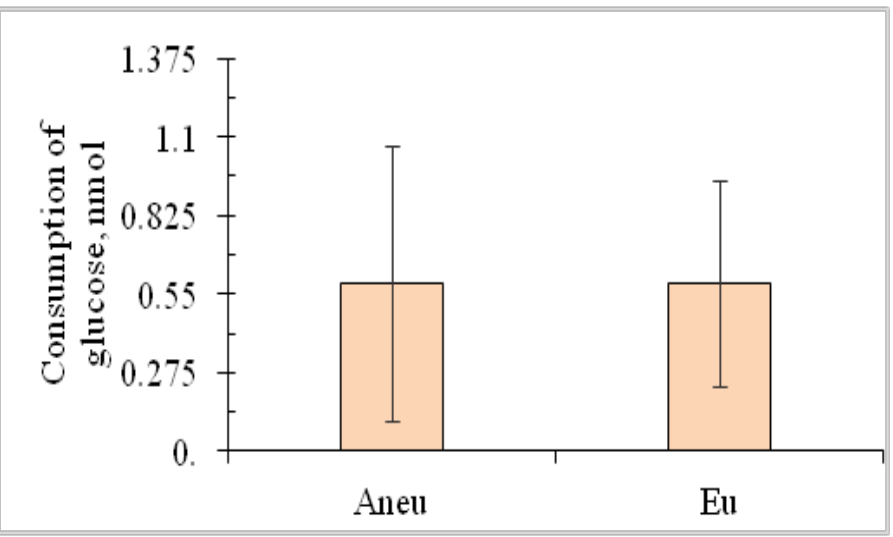

Figure I Glucose uptake by euploid and aneuploid embryos (Mann-Whitney test, $\mathrm{p}<0.05)$.

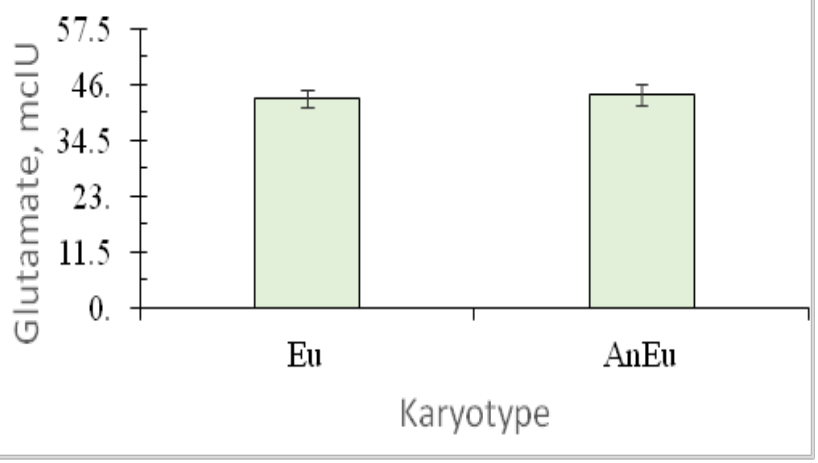

Figure 2 Glutamate uptake by euploid and aneuploid embryos (MannWhitney test, $\mathrm{p}<0.05$ ).

Average rate of glucose consumption by spent culture media for implanted embryos was 2,5 (2,0-3,4) nmol and for unimplanted embryos - 0,75 (0-1,6) nmol. Implanted euploid embryos consumed 3,4 times more glucose than that consumed unimplanted euploid embryos (Figure 3).

Consequently high level of glucose consumption out of culture media can be predictor of successful implantation of embryo.

\section{Discussion}

Metabolomics is an emerging "omics" science that has evolved from proteomics, genomics and transcriptomics. It systematically analyzes the inventory of metabolites.

Metabolomics are promising new approaches to assess embryo viability using non-invasive methods. A direct, simple and rapid analysis of spent culture media from blastocysts at the point of embryo transfer can quickly identify embryos with the best chance of achieving ongoing pregnancy. Methods like this, which take less than $20 \mathrm{~min}$ to perform, could dramatically improve the approach to embryo selection and live births.

Ray K. Iles et al. investigated 401 samples of spent culture media 
collected from embryo cultures at the time of embryo transfer, of which 136 were used to construct the predictive model. With a simple algorithm, it was possible to identify samples with the best chance of becoming an ongoing pregnancy. ${ }^{31}$

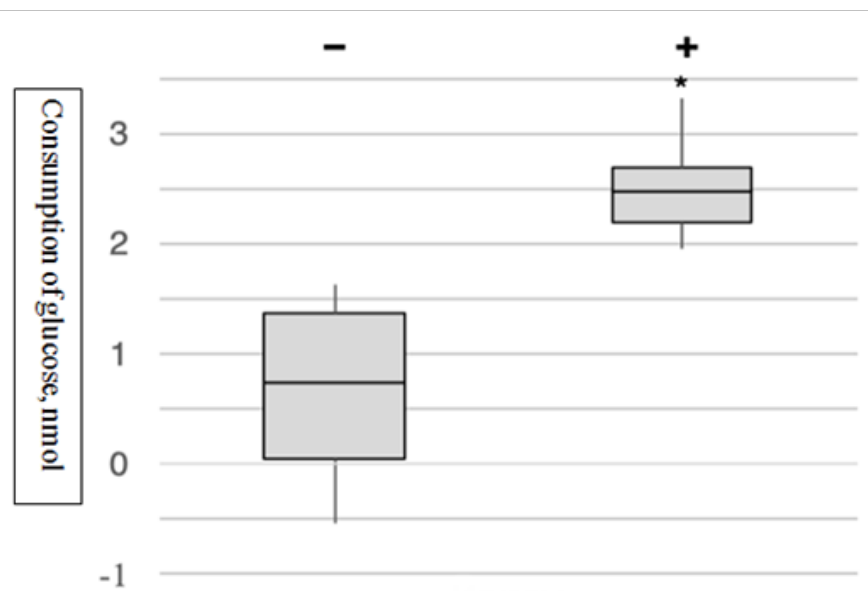

OUTCOMES

Figure 3 Glucose consumption by implanted (+) and non-implanted (-) euploid embryos 5 days of development. (The line in the middle of the "Box" is the median (50th percentile), the border is the first and third quartiles (the 25th and 75th percentile). The ends of the "whiskers" correspond to the minimum and maximum values. Extreme points (outliers) are data that go beyond the whiskers. * $-\mathrm{p}<0.05$ (Mann-Whitney test).

Ercan Baştu examined spent culture media using Raman spectroscopy from 31 women. Clinical pregnancy was predicted using Raman spectroscopy in 93\% (14/15) of clinically pregnant patients, and in $62.5 \%$ (10 out of 16 ) of clinically non-pregnant patients. Adding Raman spectroscopic analysis of spent embryo culture media revealed that this approach may predict clinical pregnancy as an adjunct to morphologic evaluation. ${ }^{32}$

Pyruvate is the favorite substrate for cleavage stage mammalian embryos include the human and is able to support the development of human blastocysts,$^{33}$ pyruvate and glucose uptake has previously been correlated with embryo viability. ${ }^{34}$

Based on that we can make a conclusion that our research is up-to-date ad necessary for development of assisted reproductive technologies.

\section{Conclusion}

1. Morphological quality of embryos in IVF program is effected by further factors: previous abortions, infertility factor,BMI, method of fertilization and sperm cell selection, ploidy of embryos in previous IVF programs with PGT/

2. Predictors of aneuploid embryos are age over 34, secondary infertility, verified external genital endometriosis stage I-II.

3. Analysis of composition of embryo culture media maid by method of metabolomic profiling can result in more specific selection of embryos for further transfer. Main predictors of successful implantation are concentration of L-Valine, L-Proline , alanyn-glutamine , Phenylpyruvic acid, b-L-Fucosa-1 and L-phenylalanine

4. Glucose consumption is not a predictor of aneuploid embryos.
Nevertheless change of glucose rate allows to reveal embryos with high potential of further implantation because it consumption 3,4 times higher than in the group that unimplanted.

\section{Acknowledgments}

None.

\section{Conflicts of interest}

Author has no conflict of interest to declare.

\section{References}

1. Kalem Z, Kalem MN, Gürgan T. Methods for endometrial preparation in frozen-thawed embryo transfer cycles. J Turk Ger Gynecol Assoc. 2016;17(3):168-172.

2. Mastenbroek S, Twisk M, Jannie van EA, et al. In vitro fertilization with preimplantation genetic screening. N Engl J Med. 2007;357(1):9-17.

3. Gardner DK, Lane M, Stevens J, et al. Fertil Blastocyst score affects implantation and pregnancy outcome: towards a single blastocyst transfer. Steril. 2000;73(6):1155-1158

4. Nasiri N, Eftekhari-Yazdi P. An overview of the available methods for morphological scoring of pre-implantation embryos in in vitro fertilization. Cell J. 2015;16(4):392-405.

5. Alpha Scientists in Reproductive Medicine and ESHRE Special Interest Group of Embryology. The Istanbul consensus workshop on embryo assessment: proceedings of an expert meeting. Hum Reprod. 2011;26(6):1270-1283

6. Capalbo A, Rienzi L, Cimadomo D, et al. Correlation between standard blastocyst morphology, euploidy and implantation: an observational study in two centers involving 956 screened 139 blastocysts. Hum Reprod. 2014;29(6):1173-1181.

7. Lintsen AM, Eijkemans MJ, Hunault CC, et al. Predicting ongoing pregnancy chances after IVF and ICSI: a national prospective study. Hum Reprod. 2007;22(9):2455-2462.

8. Franasiak JM, Forman EJ, Hong KH , et al. The nature of aneuploidy with increasing age of the female partner: a review of 15,169 consecutive trophectoderm biopsies evaluated with 135 comprehensive chromosomal screening. Fertil Steril. 2014;101(3):656-663.

9. Hodes-Wertz B, Grifo J, Ghadir S, et al. Idiopathic recurrent miscarriage is caused mostly by aneuploid embryos. Fertil Steril. 2012;98(3):675-680.

10. Stephenson MD, Awartani KA, Robinson WP. Cytogenetic analysis of miscarriages from couples with recurrent miscarriage: a case-control study. Hum Reprod. 2002;17(2):446-451.

11. Cimadomo D, Capalbo A, Ubaldi FM, et al. The impact of biopsy on human embryo developmental potential during preimplantation genetic diagnosis. Biomed Res Int. 2016;2016:7193075.

12. Echten-Arends J, Mastenbroek S, Sikkema-Raddatz B, et al. Chromosomal mosaicism in human preimplantation embryos: a systematic review. Hum Reprod. 2011;17(5):620-627.

13. Nagaoka SI, Hassold TJ, Hunt PA. Human aneuploidy: mechanisms and new insights into an age-old problem. Nat Rev Genet. 2012;13(7)493-504.

14. Munne S, Fischer J, Warner A, et al. Preimplantation genetic diagnosis significantly reduces pregnancy loss in infertile couples: a multicenter study. Fertil Steril. 2006;85(2):326-332.

15. Verlinsky Y, Cohen J, Munne S, et al. Over a decade of experience with preimplantation genetic diagnosis: a multicenter report. Fertil Steril. 2004;82(2):292-294. 
16. Moutou C, Goossens V, Coonen E, et al. ESHRE PGD Consortium data collection XII: cycles from January to December 2009 with pregnancy follow-up to October 2010. Hum Reprod. 2014;29(5):880-903.

17. Centers for Disease Control and Prevention. American Society for Assisted Reproductive Technology. Assisted Reproductive Technology National 134 Summary Report. Atlanta, GA: US Department of Health and Human Services; 2014.

18. Northrop LE, Treff NR, Levy B, et al. SNP microarray-based 24 chromosome aneuploidy screening demonstrates that cleavage-stage FISH poorly predicts aneuploidy in embryos that develop to morphologically normal blastocysts. Mol Hum Reprod. 2010;16(8):590-600.

19. Montag M, van der Ven K, Rösing B, et al. Polar body biopsy: a viable alternative to preimplantation genetic diagnosis and screening. Reprod Biomed Online. 2009;18(1):6-11.

20. Geraedts J, Collins J, Gianaroli L, et al. What next for preimplantation genetic screening? A polar body approach! Hum Reprod. 2010;25(3):575577.

21. Garrido N, Remohí J, Martínez-Conejero JA, et al. Contribution of sperm molecular features to embryoquality and assisted reproduction success. Reprod Biomed Online. 2008;17(6):855-865.

22. Cimadomo D, Capalbo A, Ubaldi FM, et al. The impact of biopsy on human embryo developmental potential during preimplantation genetic diagnosis. Biomed Res Int. 2016;2016:7193075.

23. Levin I, Almog B, Shwartz T, et al. Effects of laser polar-body biopsy on embryo quality. Fertil Steril. 2012;97(5):1085-1088.

24. Centers for Disease Control and Prevention. American Society for Assisted Reproductive Technology. Assisted Reproductive Technology National 134 Summary Report. Atlanta, GA: US Department of Health and Human Services; 2014

25. Botros L, Sakkas D, Seli E. Metabolomics and its application for noninvasive embryo assessment in IVF. Mol Hum Reprod. 2008;14(12):679-690.
26. Seli E, Botros L, Sakkas D, et al. Burns using proton nuclear magnetic resonance correlates with reproductive potential of embryos in women undergoing in vitro fertilization. Fertility and Sterility. 90:2031-2448

27. Juneau C, Franasiak J, Treff N. Challenges facing contemporary preimplantation genetic screening. Curr Opin Obstet Gynecol. 2016;28(3):151-157.

28. Seli E, Sakkas D, Scott R, et al. Noninvasive metabolomic profiling of embryo culture media using Raman and near infrared spectroscopy correlates with reproductive potential of embryos in women undergoing in vitro fertilization. Fertil Steril. 2007:88:1350-1357.

29. Scott R, Seli E, Miller K, et al. Noninvasive metabolomic profiling of human embryo culture media using Raman spectroscopy predicts embryonic reproductive potential: a prospective blinded pilot study. Fertil Steril. 2008;90:77-83.

30. Crha I, Mádr A, Musilová J, et al. The new technologies for the analytical examination of the embryonic metabolome and its prospects. Ces Gynek. 2012;77(6):502-506.

31. Iles RK, Sharara FI, Zmuidinaite R, et al. Secretome profile selection of optimal IVF embryos by matrix-assisted laser desorption ionization timeof-flight mass spectrometry. J Assist Reprod Genet. 2019.

32. Baştu E, Parlatan U, Başar G, et al. Spectroscopic analysis of embryo culture media for predicting reproductive potential in patients undergoing in vitro fertilization. Turk J Obstet Gynecol. 2017;14(3):145-150.

33. Conaghan J, Handyside AH, Winston RM, et al. Effects of pyruvate and glucose on the development of human preimplantation embryos in vitro. $J$ Reprod Fertil. 1993;99(1):87-95.

34. Wale PL, Gardner DK. Oxygen regulates amino acid turnover and carbohydrate uptake during the preimplantation period of mouse embryo development. Biol Reprod. 2012;87(1):1-8. 